\title{
Competition between Anderson localization and antiferromagnetism in correlated lattice fermion systems with disorder
}

\author{
Krzysztof Byczuk, ${ }^{1,2}$ Walter Hofstetter, ${ }^{3}$ and Dieter Vollhardt ${ }^{1}$ \\ ${ }^{1}$ Theoretical Physics III, Center for Electronic Correlations and Magnetism, \\ Institute for Physics, University of Augsburg, D-86135 Augsburg, Germany \\ ${ }^{2}$ Institute of Theoretical Physics, University of Warsaw, ul. Hoża 69, PL-00-681 Warszawa, Poland \\ ${ }^{3}$ Institut für Theoretische Physik, Johann Wolfgang Goethe-Universität, 60438 Frankfurt/Main, Germany
}

(Dated: November 6, 2018)

\begin{abstract}
The magnetic ground state phase diagram of the disordered Hubbard model at half-filling is computed in dynamical mean-field theory supplemented with the spin resolved, typical local density of states. The competition between many-body correlations and disorder is found to stabilize paramagnetic and antiferromagnetic metallic phases at weak interactions. Strong disorder leads to Anderson localization of the electrons and suppresses the antiferromagnetic long-range order. Slater and Heisenberg antiferromagnets respond characteristically different to disorder. The results can be tested with cold fermionic atoms loaded into optical lattices.
\end{abstract}

PACS numbers: 71.10.Fd, 71.27.+a, 67.85.Lm 71.30.+h

Interacting quantum many-particle systems with disorder pose fundamental challenges for theory and experiment not only in condensed matter physics $[1,2,23,4,5]$, but most recently also in the field of cold atoms in optical lattices [6, 7, 8, 9, 10]. Indeed, ultracold gases have quickly developed into a fascinating new laboratory for quantum many-body physics; see e.g. 11, 12, 13, 14, 15, 16, 17]. A major advantage of cold atoms in optical lattices is the high degree of controllability of the interaction and the disorder strength, thereby allowing a detailed verification of theoretical predictions.

In particular, these quantum many-body systems will allow for the first experimental investigation of the simultaneous presence of strong interactions and strong disorder. This very interesting parameter regime is not easily accessible in correlated electron materials. Namely, at or close to half-filling where interaction effects become particularly pronounced, strong disorder implies fluctuations (e.g., of local energies) of the order of the band-width, which usually leads to structural instabilities. These limitations are absent in the case of cold atoms in optical lattices where disorder can be tuned to become arbitrarily strong without destroying the experimental setup. Since at half filling and in the absence of frustration effects interacting fermions order antiferromagnetically, several basic questions arise: (i) How is a non-interacting, Anderson localized system at half filling affected by a local interaction between the particles? (ii) How does an antiferromagnetic insulator at half filling respond to disorder which in the absence of interactions would lead to an Anderson localized state? (iii) Do Slater and Heisenberg antiferromagnets behave differently in the presence of disorder? In this Letter we provide answers to the above questions by calculating the zero temperature, magnetic phase diagram of the disordered Hubbard model at half filling using Dynamical Mean-Field Theory (DMFT) [18, 19, 20] with a geometric average over the disorder [21, 22, 23, 24, 25] and allowing for a spin-dependence of the density of states (DOS).

Antiferromagnetic (AF) long-range order is a generic property of interacting lattice fermions with particle-hole symmetry, as exemplified by the Hubbard model at half filling with nearest-neighbor hopping on a bipartite lattice [26, 27, 28]. Such an instability is also highly relevant for current and future experiments in optical lattices [29], where the magnetic super-exchange energy scale has recently been observed in a two-component bosonic mixture [30]. The influence of disorder, e.g., due to fluctuating local potentials, on interacting quantum particles is subtle and leads to a remarkably rich phase diagram which was studied by a variety of numerical techniques 31, 32, 33, 34, 35, 36, 37]. While previous investigations yielded important insights into the properties of disordered Hubbard antiferromagnets in various regions of parameter space, a comprehensive study, where effects due to Anderson localization, genuine many-body correlations and $\mathrm{AF}$ order are treated within the same nonperturbative theoretical framework, did not yet exist. To this end we here employ the DMFT - a non-perturbative approach to correlated lattice fermions which accounts for the Mott-Hubbard metal-insulator transition (MIT) and magnetic ordering - in combination with a disorder average which is able to detect Anderson localization on the one-particle level [21, 22, 23]. Namely, by employing the geometric rather than the arithmetic average over the disorder it is possible to determine the typical local DOS 38] as a dynamical mean field within the DMFT. This approach was recently employed to calculate the paramagnetic phase diagram of the disordered Hubbard model 24] and Falicov-Kimball model [25]. Thereby it was possible to determine the MIT due to disorder (Anderson localization) and interactions (Mott-Hubbard transition), respectively, as well as the transition scenario caused by their simultaneous presence, within a unified framework. 
In the absence of frustration effects the Mott-Hubbard MIT is completely hidden by AF long-range order 19, 28]. To capture this feature it is necessary to generalize the investigation and include $\mathrm{AF}$ solutions of the Hubbard model with local disorder (Anderson-Hubbard model), whose Hamiltonian is given by

$H_{A H}=-\sum_{i j \sigma} t_{i j} a_{i \sigma}^{\dagger} a_{j \sigma}+\sum_{i \sigma} \epsilon_{i} n_{i \sigma}+U \sum_{i}\left(n_{i \uparrow}-\frac{1}{2}\right)\left(n_{i \downarrow}-\frac{1}{2}\right)$.

Here $t_{i j}$ is the amplitude for hopping between the sites $i$ and $j, U$ is the on-site repulsion, $n_{i \sigma}=a_{i \sigma}^{\dagger} a_{i \sigma}$ is the local fermion number operator with $a_{i \sigma}\left(a_{i \sigma}^{\dagger}\right)$ as the annihilation (creation) operator of a fermion with spin $\sigma$, and $\epsilon_{i}$ are random on-site energies. In the following we work with a continuous probability distribution function for $\epsilon_{i}$, i.e., $\mathcal{P}\left(\epsilon_{i}\right)=\Theta\left(\Delta / 2-\left|\epsilon_{i}\right|\right) / \Delta$, with $\Theta$ as the step function. The parameter $\Delta$ is a measure of the disorder strength. We consider a bipartite lattice with equal number of fermions and lattice sites (half-filled case). In the absence of disorder the Hamiltonian is then explicitly particle-hole symmetric.

The Anderson-Hubbard model (10) is solved within DMFT by mapping it onto single-impurity Anderson Hamiltonians with different $\epsilon_{i}$ [19, 32]. For each random on-site energy $\epsilon_{i}$, where $i$ belongs to one of the sublattices $s=\mathrm{A}$ or $\mathrm{B}$, we calculate the local Green function $G_{\sigma s}\left(\omega, \epsilon_{i}\right)$. From this quantity we obtain the geometrically averaged local DOS $\rho_{\sigma s}^{\text {geom }}(\omega)=\exp \left[\left\langle\ln \rho_{\sigma s}\left(\omega, \epsilon_{i}\right)\right\rangle\right]$ [21, 22], where $\rho_{\sigma s}\left(\omega, \epsilon_{i}\right)=-\operatorname{Im} G_{\sigma s}\left(\omega, \epsilon_{i}\right) / \pi$, and $\langle O\rangle=$ $\int d \epsilon_{i} \mathcal{P}\left(\epsilon_{i}\right) O\left(\epsilon_{i}\right)$ denotes the arithmetic average of $O\left(\epsilon_{i}\right)$. For comparison, the arithmetically averaged local DOS $\rho_{\sigma s}^{\operatorname{arith}}(\omega)=\left\langle\rho_{\sigma s}\left(\omega, \epsilon_{i}\right)\right\rangle$ is also computed. The averaged local Green function is then obtained from the Hilbert transform $G_{\sigma s}^{\alpha}(\omega)=\int d \omega^{\prime} \rho_{\sigma s}^{\alpha}\left(\omega^{\prime}\right) /\left(\omega-\omega^{\prime}\right)$, where $\alpha=$ geom (arith) denotes the geometric (arithmetic) average. The local self-energy $\Sigma_{\sigma s}^{\alpha}(\omega)$ is determined from the k-integrated Dyson equation $\Sigma_{\sigma s}^{\alpha}(\omega)=\omega-\eta_{\sigma a}^{\alpha}(\omega)-$ $1 / G_{\sigma s}^{\alpha}(\omega)$ where $\eta_{\sigma s}^{\alpha}(\omega)$ is the hybridization function of the effective Anderson Hamiltonian. The latter quantity provides the position and the resonant broadening of single-site quantum levels and may be interpreted as a molecular mean-field which describes the effect of all other sites within the DMFT. The self-consistent DMFT equations are closed by the Hilbert transform of the Green function on a bipartite lattice

$$
G_{\sigma s}^{\alpha}(\omega)=\int d \epsilon \frac{N_{0}(\epsilon)}{\left[\omega-\Sigma_{\sigma s}^{\alpha}(\omega)-\frac{\epsilon^{2}}{\omega-\Sigma_{\sigma \bar{s}}^{\alpha}(\omega)}\right]},
$$

where $N_{0}(\epsilon)$ is the non-interacting DOS and $\bar{s}$ denotes the sublattice opposite to $s$. In the following we choose a model DOS, $N_{0}(\epsilon)=2 \sqrt{D^{2}-\epsilon^{2}} / \pi D^{2}$, with bandwidth $W=2 D$, and set $W=1$. For this DOS and a bipartite lattice the local Green function and the hybridization function are connected by the simple algebraic relation

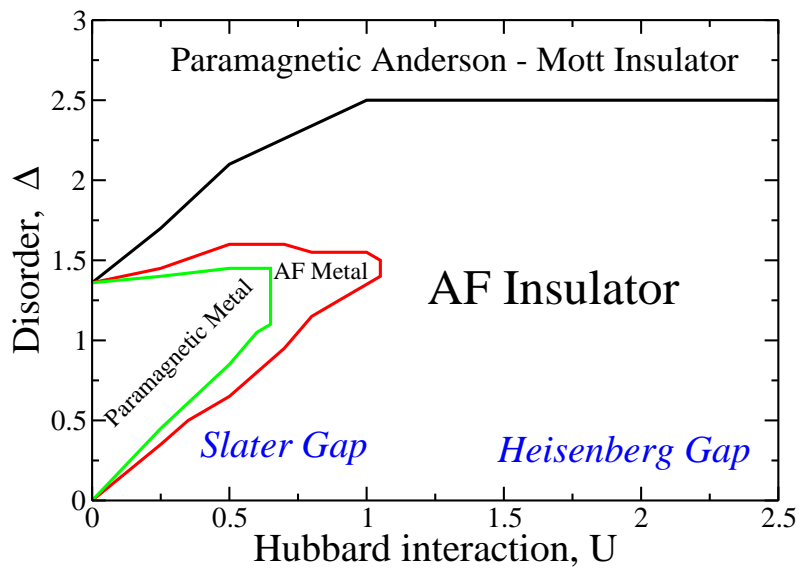

FIG. 1: Magnetic ground state phase diagram of the Anderson-Hubbard model at half-filling as calculated by DMFT with a spin resolved local DOS (see text).

$\eta_{\sigma s}^{\alpha}(\omega)=D^{2} G_{\sigma \bar{s}}^{\alpha}(\omega) / 4$ [19]. The DMFT equations are solved at zero temperature by the numerical renormalization group technique [39], which allows us to calculate the geometric or arithmetic average of the local DOS in each iteration loop.

To characterize the ground state of the Hamiltonian (11) the following quantities are computed: The local DOS $\rho_{\sigma s}^{\alpha}(\omega)$ for a given sublattice $s$ and spin direction $\sigma$, the total DOS for a given sublattice $s$ at the Fermi level $N_{s}^{\alpha}(0) \equiv \sum_{\sigma} \rho_{\sigma s}^{\alpha}(\omega=0)$, and the staggered magnetization $m_{\mathrm{AF}}^{\alpha}=\left|n_{\uparrow A}^{\alpha}-n_{\uparrow B}^{\alpha}\right|$, where $n_{\sigma s}^{\alpha}=\int_{-\infty}^{0} d \omega \rho_{\sigma s}^{\alpha}(\omega)$ is the on-site particle density on each sublattice. The possible phases of the Anderson-Hubbard model can then be classified as follows: The systems is a

i) paramagnetic metal if $N_{s}^{\text {geom }}(0) \neq 0$ and $m_{\mathrm{AF}}^{\text {geom }}=0$, ii) $\mathrm{AF}$ metal if $N_{s}^{\text {geom }}(0) \neq 0$ and $m_{\mathrm{AF}}^{\text {geom }} \neq 0$,

iii) AF insulator if $N_{s}^{\text {geom }}(0)=0$ and $m_{\mathrm{AF}}^{\text {geom }} \neq 0$ but $N_{s}^{\text {geom }}(\omega) \neq 0$ for some $\omega \neq 0$, and

iv) paramagnetic Anderson-Mott insulator if $N_{s}^{\text {geom }}(\omega)=$ 0 for all $\omega$.

The ground state phase diagram of the AndersonHubbard model (11) obtained by this classification is shown in Fig. 1. Depending on whether the interaction $U$ is weak or strong the response of the system to disorder is found to be very different. In particular, at strong interactions, $U / W \gtrsim 1$, there exist only two phases, an AF insulating phase at weak disorder, $\Delta / W \lesssim 2.5$, and a paramagnetic Anderson-Mott insulator at strong disorder, $\Delta / W \gtrsim 2.5$. The transition between these two phases is continuous. Namely, the local DOS and the staggered magnetization both decrease gradually as the disorder $\Delta$ increases and vanish at their mutual boundary (lower panel of Fig. 2). By contrast, the phase diagram for weak interactions, $U / W \lesssim 1$, has a much richer structure (Fig. 1). In particular, for weak disorder a paramagnetic metallic phase is stable. It is separated from the AF insulating phase at large $U$ by a narrow region of $A F$ 


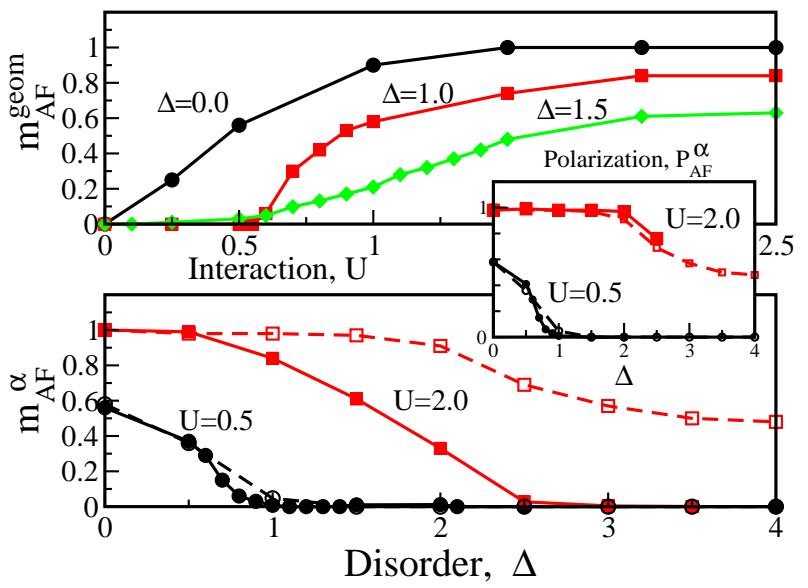

FIG. 2: Upper panel: Staggered magnetization $m_{\mathrm{AF}}^{\text {geom }}$ as a function of interaction $U$. Lower panel: $m_{\mathrm{AF}}^{\alpha}, \alpha=$ geom/arith, as a function of disorder $\Delta$. Inset: Polarization $P_{\mathrm{AF}}^{\alpha}$ as a function of disorder. Dashed lines present results obtained by arithmetic averaging.

metallic phase.

To better understand the nature of the AF phases in the phase diagram we take a look at the staggered magnetization $m_{\mathrm{AF}}^{\alpha}$. The dependence of $m_{\mathrm{AF}}^{\text {geom }}$ on $U$ is shown in the upper panel of Fig. 2 for several values of the disorder $\Delta$. In contrast to the non-disordered case a finite interaction strength $U>U_{c}(\Delta)$ is needed to stabilize the $\mathrm{AF}$ long-range order when disorder is present. The staggered magnetization saturates at large $U$ for both averages; the maximal values depend on the disorder strength. In the lower panel of Fig. 2 the dependence of $m_{\mathrm{AF}}^{\alpha}$ on the disorder $\Delta$ is shown for different interactions $U$. Only for small $U$ do the two averages yield approximately the same results. Another useful quantity is the polarization $P_{\mathrm{AF}}^{\alpha}=m_{\mathrm{AF}}^{\alpha} / I^{\alpha}$, where $I^{\alpha}=\int_{-\infty}^{+\infty} \sum_{\sigma s} \rho_{\sigma s}^{\alpha}(\omega) d \omega / 2$ is the total spectral weight of $\rho_{\sigma s}^{\alpha}(\omega)$. It allows one to investigate the contribution of the point-like spectrum of the Anderson localized states to the magnetization. This provides important information about the spectrum since with increasing disorder more and more one-particle states of the many-body system are transferred from the continuous to the point-like spectrum. For weak interactions $(U=0.5)$ the decrease of the polarization with increasing disorder $\Delta$ obtained with geometric or arithmetic averaging is the same (see inset in Fig. 21). Since arithmetic averaging does not treat states from the pointlike spectrum correctly, the decrease of $m_{\mathrm{AF}}^{\alpha}$ (which is also the same for the two averages, see lower panel of Fig. 2) must be attributed to disorder effects involving only the continuous spectrum. At larger $U$ the polarization is constant up to the transition from the AF insulator to the paramagnetic Anderson-Mott insulator. In the latter phase the polarization is undefined, because the continuous spectrum does not contribute to $I_{\mathrm{AF}}^{\mathrm{geom}}$.

The AF metallic phase is long-range ordered, but there
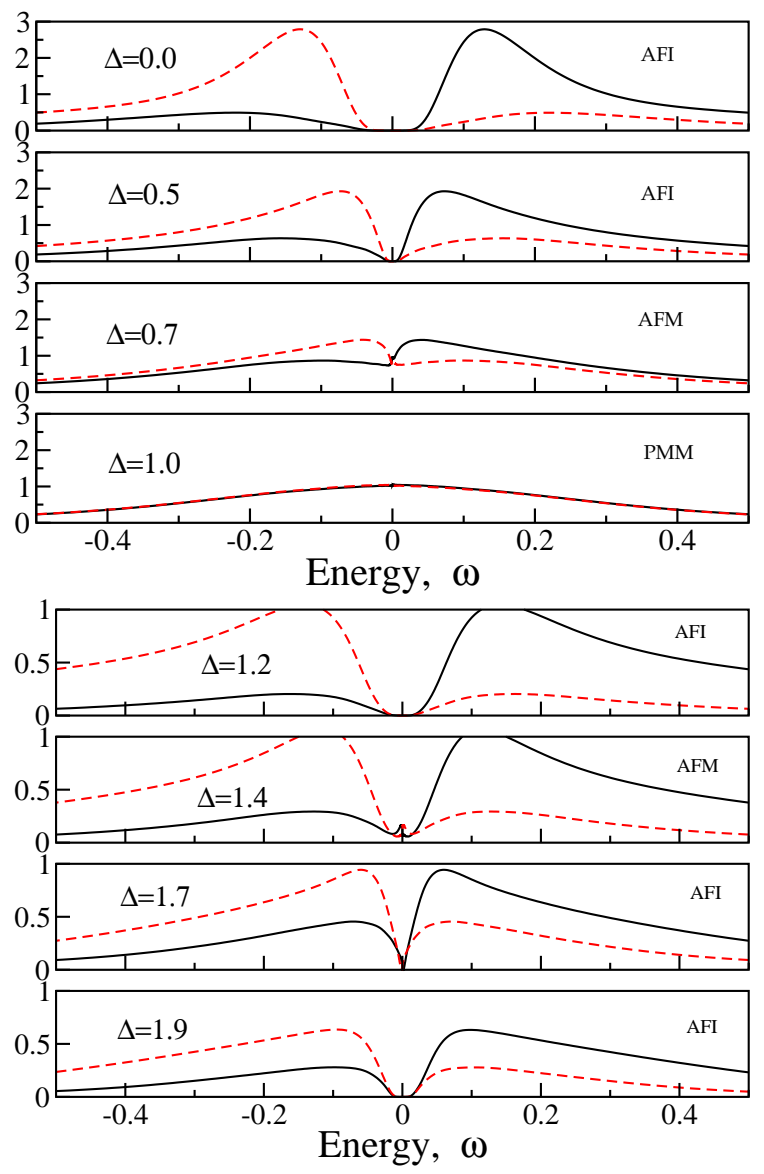

FIG. 3: Typical local DOS as a function of disorder $\Delta$ for interaction $U=0.5$ (upper panel) and $U=1.0$ (lower panel). Solid and dashed lines represent opposite spin directions.

is no gap since the disorder leads to a redistribution of spectral weight. In Fig. 3 the local DOS in the vicinity of the transitions between the paramagnetic metal, the $\mathrm{AF}$ insulator and the AF metal at $U=0.5$ (upper panel), and the transitions between the $\mathrm{AF}$ insulator, the $\mathrm{AF}$ metal and back into the $\mathrm{AF}$ insulator at $U=1.0$ (lower panel) are shown. The paramagnetic metal, where $\rho_{\sigma s}^{\alpha}(\omega)=$ $\rho_{\sigma \bar{s}}^{\alpha}(\omega)$, is seen to be stable only for weak interactions.

In the absence of disorder the AF insulating phase has a small ("Slater") gap at $U / W<1$ and a large ("Heisenberg") gap at $U / W>1$. These limits can be described by perturbation expansions in $U$ and $1 / U$ around the symmetry broken state of the Hubbard and the corresponding Heisenberg model, respectively. In agreement with earlier studies [40] our results for $m_{\mathrm{AF}}$ (upper panel of Fig. 21) show that there is no sharp transition between these limits, even when disorder is present. This may be attributed to the fact that both limits are described by the same order parameter. However, the phase diagram (Fig. 1) shows that the two limits can be distinguished by their overall response to disorder. Namely, the reentrance of the $\mathrm{AF}$ metallic phase at $\Delta / W \gtrsim 1$ occurs only 
within the Slater AF insulating phase.

The magnetic structure of the Anderson-Mott insulator cannot be determined by the method used here since it describes only the continuous part of the spectra and not the point spectrum. However, only the paramagnetic solution should be expected to be stable because the kinetic exchange interaction responsible for the formation of the AF metal is suppressed by the disorder. This does not exclude the possibility of Griffiths phase-like AF domains [41].

It is interesting to note that even the DMFT with an arithmetic average finds a disordered AF metal [32, 34]. However, the arithmetically averaged local DOS incorrectly predicts both the paramagnetic metal and the AF metal to remain stable for arbitrarily strong disorder. Only a computational method which is sensitive to Anderson localization, such as the DMFT with geometrically averaged local DOS employed here, is able to detect the suppression of the metallic phase for $\Delta / W \gtrsim 1.5$ and the appearance of the paramagnetic Anderson-Mott insulator at large disorder $\Delta$ already on the one-particle level.

In conclusion, we computed the ground state phase diagram of the Anderson-Hubbard model at half filling within a non-perturbative approach which can treat interactions and disorder of arbitrary strength and is sensitive to Anderson localization on the one-particle level. For low disorder and weak interactions paramagnetic and antiferromagnetic metallic phases become stable, with a reentrant behavior of the latter phase. Slater and Heisenberg antiferromagnets can be distinguished by their very different response with respect to disorder. Experiments with cold fermionic atoms loaded into optical lattices will be able to test these predictions and check the accuracy of the theoretical approach employed here.

We thank R. Bulla for useful discussions. This work was supported in part by the Sonderforschungsbereich 484 and the Forschergruppe FOR 801 of the Deutsche Forschungsgemeinschaft.

[1] N. F. Mott, Proc. Phys. Soc. A 62, 416 (1949); MetalInsulator Transitions, 2nd edn. (Taylor and Francis, London 1990).

[2] P. A. Lee and T. V. Ramakrishnan, Rev. Mod. Phys. 57, 287 (1985).

[3] B.L. Altshuler and A.G. Aronov, in Electron-Electron Interactions in Disordered Systems, eds. M. Pollak and A.L. Efros (North-Holland, Amsterdam, 1985), p.1.

[4] D. Belitz and T. R. Kirkpatrick, Rev. Mod. Phys. 66, 261 (1994).

[5] E. Abrahams, S.V. Kravchenko, and M.P. Sarachik, Rev. Mod. Phys. 73, 251 (2001).

[6] M. Lewenstein, et al., Adv. Phys. 56, 243 (2007).

[7] L. Fallani et al., Phys. Rev. Lett. 98, 130404 (2007).

[8] J. Billy et al., Nature 453, 891 (2008).
[9] G. Roati et al., Nature 453, 895 (2008).

[10] M. White et al., arXiv:0807.0446.

[11] I. Bloch, J. Dalibard, and W. Zwerger, Rev. Mod. Phys. 80, 885 (2008).

[12] D. Jaksch et al., Phys. Rev. Lett. 89, 040402 (2002).

[13] M. Greiner et al., Nature 415, 39 (2002).

[14] W. Hofstetter et al., Phys. Rev. Lett. 89, 220407 (2002).

[15] M. Köhl et al., Phys. Rev. Lett. 94, 080403 (2005).

[16] S. Ospelkaus et al., Phys. Rev. Lett. 96, 180403 (2006); K. Günter et al., Phys. Rev. Lett. 96, 180402 (2006).

[17] R. Jördens et al., Nature 455, 204 (2008); U. Schneider et al., preprint arXiv:0809.1464.

[18] W. Metzner and D. Vollhardt, Phys. Rev. Lett. 62, 324 (1989).

[19] A. Georges, G. Kotliar, W. Krauth, and M. J. Rozenberg, Rev. Mod. Phys. 68, 13 (1996).

[20] G. Kotliar and D. Vollhardt, Physics Today 57, 53 (2004).

[21] V. Dobrosavljević and G. Kotliar, Phys. Rev. Lett. 78, 3943 (1997).

[22] V. Dobrosavljević, A. A. Pastor, and B. K. Nikolić, Europhys. Lett. 62, 76 (2003).

[23] G. Schubert et al., in High Performance Computing in Science and Engineering Garching 2004, Eds. A. Bode, F. Durst, p. 237-250, (Springer Verlag, 2005).

[24] K. Byczuk, W. Hofstetter, and D. Vollhardt, Phys. Rev. Lett. 94, 056404 (2005).

[25] K. Byczuk, Phys. Rev. B 71, 205105 (2005).

[26] D. R. Penn, Phys. Rev. 142, 350 (1966); W. Langer, M. Plischke, and D. Mattis, Phys. Rev. Lett. 23,1448 (1969).

[27] M. Jarrell, Phys. Rev. Lett. 69,168 (1992).

[28] Th. Pruschke, Prog. Theo. Phys. Suppl. 160, 274 (2005).

[29] F. Werner at al., Phys. Rev. Lett. 95, 056401 (2005);

A. Koetsier et al., Phys. Rev. A 77, 023623 (2008); K. Eckert at al., Nature Phys. 4, 50 (2008); M. Snoek et al., New J. Phys. 10, 093008 (2008).

[30] S. Fölling et al., Nature 448, 1029 (2007), S. Trotzky et al., Science 319, 295 (2008).

[31] M. A. Tusch and D. E. Logan, Phys. Rev. B 48, 14843 (1993).

[32] M. Ulmke, V. Janiš, and D. Vollhardt, Phys. Rev. B 51, 10411 (1995).

[33] P.J.H. Denteneer, M. Ulmke, R.T. Scalettar, and G.T. Zimanyi, Physica A 251, 162 (1998).

[34] A. Singh, M. Ulmke, and D. Vollhardt, Phys. Rev. B 58, 8683 (1998).

[35] D. Heidarian and N. Trivedi, Phys. Rev. Lett. 93, 126401(2004).

[36] B.M. Andersen, P.J. Hirschfeld, A.P. Kampf, and M. Schmid, Phys. Rev. Lett. 99, 147002 (2007).

[37] M.E. Pezzoli, F. Becca, M. Fabrizio, G. Santoro, arXiv:0808.0877

[38] P. W. Anderson, Phys. Rev. 109, 1492 (1958).

[39] K. Wilson, Rev. Mod. Phys. 47, 773 (1975); R. Bulla, A. C. Hewson, and Th. Pruschke, J. Phys.: Condens. Matter 10, 8365 (1998); W. Hofstetter, Phys. Rev. Lett. 85, 1508 (2000); R. Bulla, T. A. Costi, and D. Vollhardt, Phys. Rev. B 64, 045103 (2001).

[40] Th. Pruschke and R. Zitzler, J. Phys.: Condens. Matter 15, 7867 (2003); R. Peters and Th. Pruschke, arXiv:0809.2689

[41] R.B. Griffiths, Phys. Rev. Lett. 23, 17 (1969); V. Dobrosavljevic, E. Miranda, Phys. Rev. Lett. 94, 187203 (2005). 